\title{
BERICHT VOM 9. MISSING LINIK EBOOK-TAG (BREMEN, 30./31. MÄRZ 2017)
}

\section{von Birgit Bittner \& Christof Capellaro}

Am 30. und 31. März 2017 richtete die Firma Missing Link in den Tagungsräumen der Kunsthalle Bremen den Bremer eBook-Tag aus. Im inzwischen schon neunten Jahr seines Bestehens hat sich dieser zu einem sehr attraktiven Forum des Austausches zwischen Buchhändlerlnnen, Verlagsvertreterlnnen und BibliothekarInnen zum Thema eBooks und anderen elektronischen Bibliotheksprodukten entwickelt. Dementsprechend waren diesmal auch mehr als 90 Gäste aus Deutschland, Österreich und der Schweiz der Einladung nach Bremen gefolgt.

Nach kurzer Begrüßung durch die Gastgeber Klaus Tapken und Branka Felba (Missing Link) betonte Frau Prof. Dr. Eva Quante-Brandt, Bremer Senatorin für Wissenschaft, Gesundheit und Verbraucherschutz, in ihrem Grußwort den immer weiter fortschreitenden Übergang von gedruckten zu elektronischen Formen der Literaturversorgung. Mit den sog. DEAL-Lizenzen und dem Referentenentwurf zur Reform des Urheberrechtsgesetzes sprach die Senatorin aber auch zwei ganz konkrete Themen an, die die bibliothekarische, verlegerische und wissenschaftspolitische Debatte in der Bundesrepublik Deutschland zurzeit prägen.

Der erste Fachvortrag des Tages stand unter dem Titel „Open Access in den Geisteswissenschaften - zweitklassiges Publizieren oder Chance zur Marktveränderung?“ und wurde von Margo Bargheer gehalten, die beim Universitätsverlag Göttingen tätig ist, der pro Jahr etwa 50 wissenschaftliche Buchpublikationen betreut, die allesamt Open Access erscheinen. Ein Teil der Titel wird zusätzlich auch in gedruckter Form herausgegeben, je nach den Bedürfnissen der Leserschaft. Bargheer skizzierte zunächst den Publikationsprozess und die damit zusammenhängende Wertschöpfungskette am Beispiel der Geisteswissenschaften. Hierbei hob sie hervor, dass unterschiedliche Akteure als einzelne Glieder dieser Kette auch unterschiedliche Ziele verfolgen: Während für die publikationswilligen Wissenschaftler Prestige- und Reputationsgewinn (Impactfactor) im Vordergrund stehen, ist das Hauptziel von Großverlagen die Profitmaximierung. Durch Open Access werden neue Akteure Teil der Publikations- und Wertschöpfungs- 
kette, so etwa Anbieter wie ubiquity press, ein kommerzielles Unternehmen, das technische Plattformen für Open-Access-Verlage bereitstellt. An einigen Beispielen aus der Qualitätspresse demonstrierte Bargheer dann, dass der Open-Access Gedanke teils absichtlich missverstanden wird. Vor diesem Hintergrund plädierte sie unter dem Motto „Probieren statt Polemisieren" dafür, Open-Access-Modelle praktisch zu erproben und an ihrem tatsächlichen Wirkungsgrad zu messen. Dieser Wirkungsgrad ist umso höher, je besser Open-Access-Veröffentlichungen von potentiellen Interessenten gefunden werden können. Aus diesem Grund kommt Metadaten für Open-Access-Veröffentlichungen große Bedeutung zu. Die Kurzvorstellung einiger für die Geisteswissenschaften besonders interessanter Open-Access-Initiativen sowie der dahinterstehenden Geschäftsmodelle (OAPEN, OpenEdition, Knowledge Unlatched, Open Humanities Press, punctum books, Open Library of Humanities) rundeten den Vortrag ab.

In dem Vortrag „Urheberrecht und Wissenschaftsschranke“ ging dann Prof. Dr. Christian Sprang, Justiziar des Börsenvereins des Deutschen Buchhandels, auf den derzeit heftig umstrittenen Referentenentwurf zur Änderung des deutschen Urheberrechtsgesetzes ein. Sprang erläuterte zunächst den Ablauf des Verfahrens und das Prinzip der sog. Schrankenregelungen im Urheberrecht. Hier sei eine deutliche Tendenz ablesbar, dass Vergütungen nicht mehr individuell, sondern über Verwertungsgesellschaften ausgeschüttet werden. Als Ausnahmetatbestände lassen Schrankenregelungen bestimmte Formen von Werknutzungen im Kontext von Forschung und Lehre zu (z.B. Zitieren in wissenschaftlichen Veröffentlichungen, Kopieren für den Unterrichtsgebrauch, Digitalisieren und Wiedergabe des Digitalisats an Leseplätzen von Bibliotheken), ohne dass es einer Zustimmung des Rechteinhabers (Autorln, Verlag) bedarf. Aus Sicht des Buchhandels beklagte Sprang, dass schon die derzeit im deutschen Urheberrechtsgesetz festgeschriebenen Schrankenregelungen zu weitreichend seien. Das Ziel des vorliegenden Referentenentwurfs, noch weitere Ausnahmetatbestände hinzuzufügen (u.a. Text und Data-Mining für wissenschaftliche Zwecke, E-Book-Ausleihe für Öffentliche Bibliotheken, gesetzliche Erlaubnis für Hochschulen, bis zu $25 \%$ eines Lehrbuchs digital bereitzustellen), lehne der Börsenverein entschieden ab.

Am Nachmittag bot ein World Cafe den anwesenden Bibliothekarlnnen, Verlagsvertreterlnnen und Buchhändlerlnnen Gelegenheit, sich in kleinen moderierten Gesprächsgruppen mit unterschiedlichen Aspekten der Erwerbung und Bereitstellung von eBooks auseinanderzusetzen. Das Spektrum reichte hier von der Bestellabwicklung, der Bewerbung und Metadatenversorgung in den Bibliotheken bis zu Administrationstools auf 
Verlagsseite. Alternativ zur Teilnahme am World Cafe wurde von Roland Rehberger (ExLibris) und Margit Breitkopf (UB Mannheim) ein fachlicher Austausch rund um das Bibliotheksverwaltungssystem ALMA angeboten.

Nach dem offiziellen Teil des Tages standen eine Führung durch den 600jährigen Bremer Ratskeller (Sitz und Weinlager nicht nur eines der ältesten, sondern auch weltweit größten Zwischenhändlers für deutsche Weine) und ein gemeinsames Abendessen auf dem Programm.

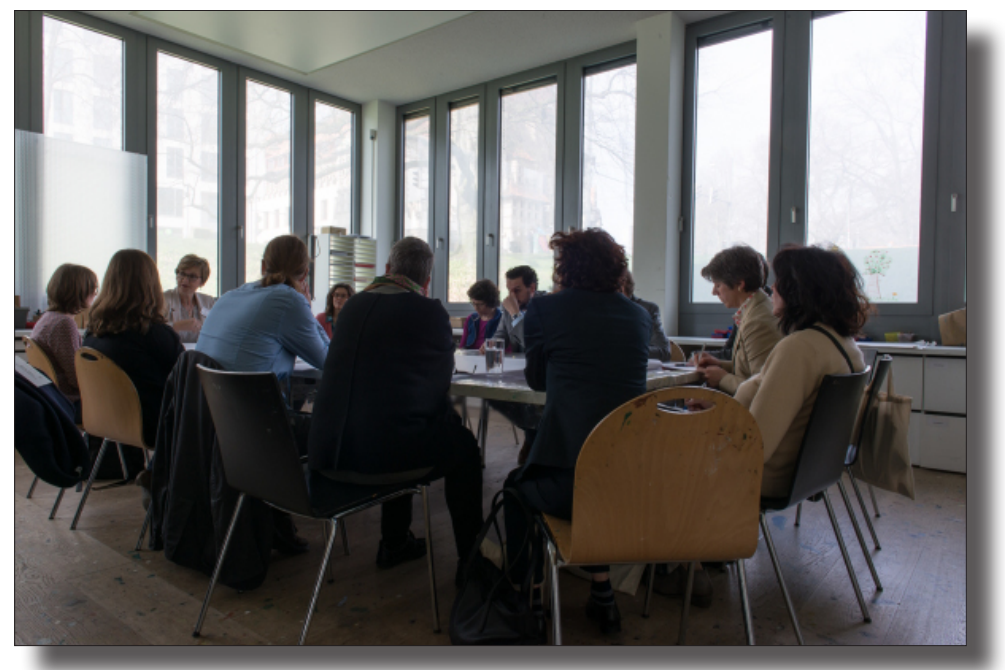

Abb. 1: Blick ins „WorldCafe“ / Tisch 1 - eBookbestellung (Foto: Missing Link)

Den Anfang des zweiten Konferenztages machte ein Vortrag von Alex Jenner (ProQuest) unter dem Titel „Approaching the Brave New World of Ebook Central: die Migration von MyiLibrary und mehr". Mehrere bislang eigenständige E-Book-Plattformen unter dem Dach von ProQuest werden seit 2015/2016 zur gemeinsamen Plattform Ebook Central zusammengeführt bzw. in diese integriert. Nachdem im Jahr 2016 schon die Plattformen EBL und Ebrary in Ebook Central aufgegangen sind, ist für 2017 die Migration von MyiLibrary nach Ebook Central geplant. Jenner erläuterte den Zeitplan und die einzelnen Stufen dieses Migrationsprozesses, der sich überaus aufwändig gestaltete, da neben den eBooks beispielsweise auch Usage-Daten und Profilinformationen der einzelnen Bibliotheken nach Ebook Central überführt werden müssen. Jenner stellte außerdem dar, welche Auswirkungen die Migration auf laufende PDA-Projekte bisheriger MyiLibrary Kunden haben wird und in welchen Punkten sich die angebotenen Erwerbungs- und Zugangsmodelle von MyiLibrary und Ebook Cen- 
tral unterscheiden (u.a. Einführung einer neuen Erwerbungsoption ,NonLinear-Lending). In der anschließenden Diskussions- und Fragerunde stieß insbesondere Jenners Ankündigung auf Interesse, dass über Ebook Cetral neben herkömmlichen PDA- künftig auch EBS-Modelle angeboten werden können. Von mehreren Bibliothekarlnnen kritisch angesprochen wurde die Struktur der Zugangslinks auf E-Book-Central (keine einheitlichen, sondern institutionsspezifische Links, daher derzeit auch kein Einsatz stabiler Links nach gängigen Industriestandards wie z.B. URNs). Jenner versprach, zumindest das Konzept der bibliotheksspezifischen Zugangslinks in den nächsten Monaten ProQuest-intern einer kritischen Revision zu unterziehen.

Im folgenden Vortrag berichtete Mario Hütte (FH Dortmund) über erste praktische Erfahrungen mit Ebook Central an einer Fachhochschulbibliothek. Dem massiven Anstieg der eBook-Nutzung wird auch in der Erwerbungspolitik der Bibliothek Rechnung getragen, unter anderem durch die Ergänzung im hauseignen Wunschbuchformular, dass die Anschaffung eines eBooks bevorzugt wird. Ein direkter Vergleich zwischen MyiLibrary und Ebook Central fällt für die FH Dormund eindeutig zugunsten von Ebook Central aus, da dort v.a. mehr deutschsprachige Titel angeboten werden und die Freischaltdauer wesentlich kürzer ist. Die Nutzungsmodalitäten von EBC erweitern sich durch die persönliche Nutzerregistrierung und durch eine automatische Upgradefunktion der Parallelzugriffsmöglichkeit kommt es zu keinen Denials.

Kaum ein anderes Segment im Buchmarkt erlebt durch die Digitalisierung einen so starken Wandel wie das Lehrbuch. Aus diesem Grund wird dem Thema Lehrbuch beim Bremer eBook-Tag besondere Aufmerksamkeit geschenkt. Dass dieser Anspruch auch heuer in gelungener Weise eingelöst werden konnte, war u.a. auch dem Vortrag von Martin Kruse (Missing Link) zu verdanken, der mit Kortext eine innovative Plattform für elektronische Lehrbuchinhalte vorstellte (über 700 Verlage im Angebot). Kortext macht einmal erworbene digitale Lehrbücher zugänglich, bietet diverse Personalisierungs- und Sharingfunktionen und unterstützt die Arbeit mit digitaler Literatur in Seminar- oder Lerngruppen. Kortext richtet sich vorrangig an Endkunden, die über die Plattform im Preis reduzierte „Textbooks" erwerben, doch können auch von der betreffenden Universitätsbibliothek lizenzierte Inhalte eingebunden werden.

Kruse berichtete, dass Kortext aus Großbritannien kommt, wo es gezielt von Universitäten eingesetzt wird, die in einem starken Konkurrenzverhältnis zueinanderstehen, was die Rekrutierung zahlungskräftiger Studierender betrifft. Diese Universitäten werben u.a. damit, dass jeder neue Studierende ein Tablet erhält, auf dem die Kortext-App mit verschiedenen digitalen 
Lehrbüchern des gewählten Faches schon vorinstalliert ist.

Im Anschluss wurden die Ergebnisse aus dem World Cafe vom Vortrag präsentiert. Diese lassen sich dahingehend zusammenfassen, dass Bibliothekarlnnen vielfältige Add-On-Services vom Verlagswesen und Buchhandel wünschen, die sie bei der Erwerbung (Budgetübersicht), Bereitstellung (Freischaltinformationen), Erschließung und Bewerbung von E-Books unterstützten. Gleichzeitig möchten die Bibliothekarlnnen aber durch die Nutzung solcher Services so wenig wie möglich in ihrer Handlungsautonomie eingeschränkt werden. Auch sollen die entsprechenden Services (z.B. Admintools) möglichst einfach nutzbar und über verschiedene Plattformen/Verlage hinweg standardisiert sein. Im Rahmen des World Cafes besonders häufig angesprochen wurde das Thema Metadaten für E-Books. Hier herrscht nach Meinung vieler Teilnehmerlnnen noch erheblicher Optimierungsbedarf, v.a. in Bezug auf Qualität (Normdatenanreicherung) und Bereitstellungsgeschwindigkeit durch Verlage und Bibliotheksverbünde. Gerade in diesem Zusammenhang wurde deutlich, wie wichtig der Austausch zwischen Bibliothekarlnnen, Vertrerterlnnen des Buchhandels und des Verlagswesens im Rahmen von Veranstaltungen wie dem Bremer E-Book-Tag ist.

Die Perspektive von WissenschaftlerInnen als AbnehmerInnen bibliothekarischer Informationsdienstleistungen brachte schließlich Prof. Dr. Armin Varmaz (Professor für Betriebswirtschaftslehre an der Hochschule Bremen) mit dem Vortrag „Welche Medien und Tools nutzen Wissenschaftler heute in der Forschung und Lehre wirklich?“ ein. Varmaz berichtete von der eigenen Herangehensweise bei der Informationssuche sowie über Rückmeldungen anderer Professorlnnen an Bremer Hochschulen, die er zu diesem Zweck befragt hatte. Als Ergebnis strich er heraus, dass sich die Arbeitsprozesse von Wissenschaftlerlnnen durch neue elektronische Angebote, die einzelne Prozessschritte unterstützen können, stark gewandelt haben (Kommunikationsplattformen und andere Collaboration-Tools, Literaturverwaltungsprogramme, High-Impact-Journal-Finder). Für viele Wissenschaftlerlnnen sei heute Google Scholar der primäre Ausgangspunkt bei der Informationssuche. Daneben spielten fachspezifische oder durch die Bibliothek vermittelte Angebote wie Verbundkataloge und Zitationsdatenbanken aber immer noch eine große Rolle. Das Serviceangebot der Bibliotheken sei insgesamt allerdings noch ausbaufähig. Varmaz wünschte sich hier vor allem mehr - möglichst prominent verlinkte - Videotutorials, die in die Nutzung spezifischer Fachdatenbanken sowie in die Bibliotheksbenutzung generell einführen. In seiner Eigenschaft als Wirtschaftswissenschaftler beklagte Varmaz, dass einschlägige Fakteninformation (Firmendaten, Finanzinformationen) durch Downloadbeschränkungen kommerzieller Anbieter 
und mangelnde Standardisierung bei nicht-kommerziellen Angeboten für die Forschung nur schwer nutzbar seien.

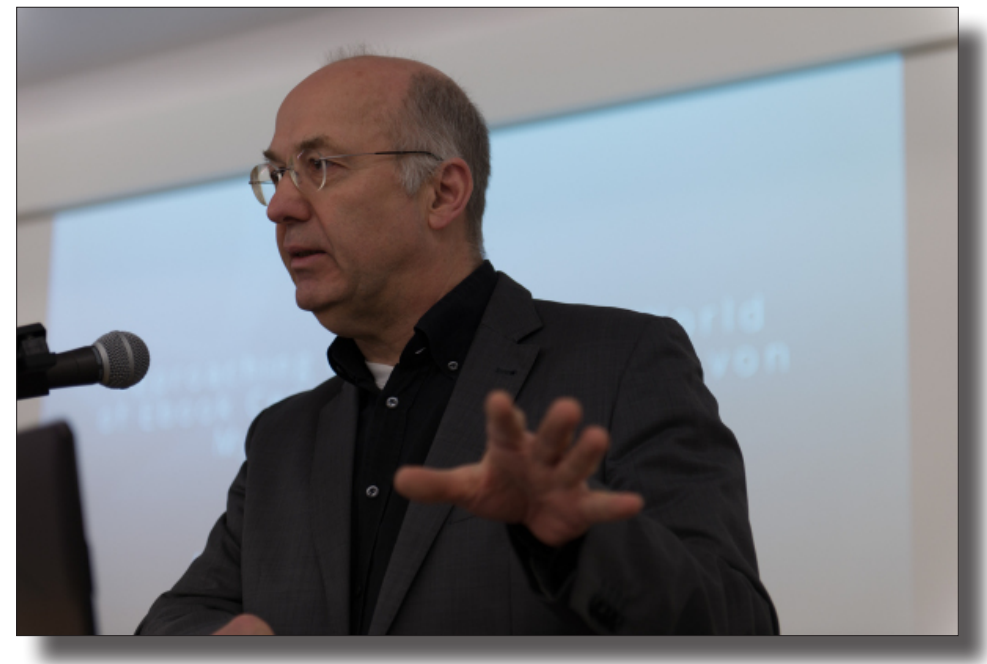

Abb. 2: Vortrag von Klaus Tapken (Foto: Missing Link)

Abschließend stellten Branka Felba und Klaus Tapken (Missing Link) aktuelle Angebote und Marktentwicklungen im eBook-Bereich vor, wobei auch hier der Schwerpunkt auf elektronischen Lehrbuchangeboten lag.

Zum Ausklang der höchst informativen Veranstaltung wurde für kunstinteressierte Teilnehmer eine Führung durch die Ausstellung „Franz Radziwill und Bremen “ in der Kunsthalle angeboten, bei der man diese wunderschöne Stadt durch die Augen des Künstlers Radziwill betrachten konnte.

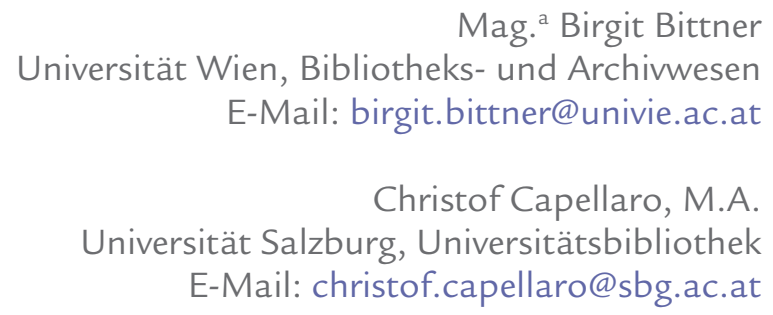

DOI: http://doi.org/10.31263/voebm.v71i1.1884

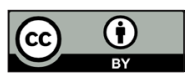

